\title{
Non-destructive electrochemical graphene transfer from reusable thin-film catalysts
}

\author{
Pizzocchero, Filippo; Jessen, Bjarke Sørensen; Whelan, Patrick Rebsdorf; Kostesha, Natalie; Lee, \\ Sunwoo; Buron, Jonas Christian Due; Petrushina, Irina; Larsen, Martin B.; Greenwood, Paul; Cha, Wu \\ Joon
}

Total number of authors:

15

Published in:

Carbon

Link to article, DOI:

10.1016/j.carbon.2014.12.061

Publication date:

2015

Document Version

Peer reviewed version

Link back to DTU Orbit

Citation (APA):

Pizzocchero, F., Jessen, B. S., Whelan, P. R., Kostesha, N., Lee, S., Buron, J. C. D., Petrushina, I., Larsen, M. B., Greenwood, P., Cha, W. J., Teo, K., Jepsen, P. U., Hone, J., Bøggild, P., \& Booth, T. (2015). Non-destructive electrochemical graphene transfer from reusable thin-film catalysts. Carbon, 85, 397-405.

https://doi.org/10.1016/j.carbon.2014.12.061

\section{General rights}

Copyright and moral rights for the publications made accessible in the public portal are retained by the authors and/or other copyright owners and it is a condition of accessing publications that users recognise and abide by the legal requirements associated with these rights.

- Users may download and print one copy of any publication from the public portal for the purpose of private study or research.

- You may not further distribute the material or use it for any profit-making activity or commercial gain

- You may freely distribute the URL identifying the publication in the public portal 


\section{Non-destructive Electrochemical Graphene Transfer from Reusable Thin-Film}

\section{Catalysts}

Filippo Pizzocchero ${ }^{\dagger}$, Bjarke S. Jessen ${ }^{\dagger, \S}$, Patrick R. Whelan ${ }^{\dagger}$, Natalie Kostesha ${ }^{\dagger}$, Sunwoo Lee', Jonas D. Buron ${ }^{\dagger,}$, , Irina Petrushina" , Martin B. Larsen ${ }^{\dagger}$, Paul Greenwood", Wu Joon Cha ${ }^{\natural}$, Ken Teo', Peter U. Jepsen ${ }^{\ddagger}$, James Hone $\urcorner$, Peter Bøggild ${ }^{\dagger, \S}$, Timothy J. Booth ${ }^{\dagger, *}$

${ }^{\dagger}$ DTU Nanotech, Technical University of Denmark, Ørsteds Plads, 345E, Kgs. Lyngby, 2800

Denmark,

${ }^{\S}$ Center for Nanostructured Graphene (CNG), Technical University of Denmark, 2800 Kgs.

Lyngby, Denmark

'Department of Electrical Engineering, Columbia University, 10027 New York, USA

${ }^{\urcorner}$Department of Mechanical Engineering, Columbia University, 10027 New York, USA

${ }^{\ddagger}$ DTU Photonics, Technical University of Denmark, Ørsteds Plads, 343, Kgs. Lyngby, 2800

Denmark

"DTU Energy Conversion, Technical University of Denmark, Kemitorvet 207, Kgs. Lyngby, 2800

Denmark

"AIXTRON Ltd, Buckingway Business Park, Anderson Road, Swavesey, Cambridge CB24 4FQ,

United Kingdom

*Corresponding author: tim.booth@nanotech.dtu.dk - +45 45256355 


\begin{abstract}
We demonstrate an electrochemical method - which we term oxidative decoupling transfer (ODT) for transferring chemical vapor deposited graphene from physically deposited copper catalyst layers. This copper oxidation-based transfer technique is generally applicable to copper surfaces, and is particularly suitable where the copper is adhered to a substrate such as oxidized silicon. Graphene devices produced via this technique demonstrate 30\% higher mobility than similar devices produced by standard catalyst etching techniques. The transferred graphene films cover more than $94 \%$ of target substrates - up to $100 \mathrm{~mm}$ diameter films are demonstrated here - and exhibit a low Raman D:G peak ratio and a homogenous and continuous distribution of sheet conductance mapped by THz time-domain spectroscopy. By applying a fixed potential of $-0.4 \mathrm{~V}$ vs. an $\mathrm{Ag} / \mathrm{AgCl}$ reference electrode - significantly below the threshold for hydrogen production by electrolysis of water - we avoid the formation of hydrogen bubbles at the graphene-copper interface, preventing delamination of thin sputtered catalyst layers from their supporting substrates. We demonstrate the reuse of the same growth substrate for five growth and transfer cycles and prove that this number is limited by the evaporation of $\mathrm{Cu}$ during growth of graphene. This technique therefore enables the repeated use of the highest crystallinity and purity substrates without undue increase in cost.
\end{abstract}

Keywords: CVD, wafer scale, G-FET, electrochemistry, intercalation, copper oxidation 


\section{Introduction}

The growth of graphene by chemical vapor deposition on copper catalyst layers is a promising route for the scalable production of industrially relevant quantities of high crystal quality monolayer graphene [1, 2]. Techniques for the transfer of the graphene from metallic catalyst layers to substrates of interest - such as oxidized silicon or flexible polymers - form an integral part of the production process; but maintaining the quality of these single-atom thick layers during delamination from their parent catalyst substrates and subsequent adhesion to a target substrate is challenging. This is particularly the case if the catalyst needs to be reused. Published techniques for the transfer of graphene from growth substrates usually involve the complete destruction of the catalyst layer by dissolution with chemical etchants such as ferric chloride or ammonium persulphate [3-5]. In an industrial context such a process would significantly increase the costs associated with the production of graphene. In particular, dissolution precludes the reuse of catalyst layers where an investment has been made in optimizing the catalyst with respect to purity, grain size, flatness, composition or other desirable characteristics, effectively discouraging real development in advanced catalyst materials. A transfer technique that preserves such an optimized layer between growths would enable the repeated production of high quality graphene, with potentially high consistency if this transfer technique does not impair catalyst performance.

One such technique has been demonstrated by Wang et al. [6] - the authors showed that application of a reducing potential of $-5 \mathrm{~V}$ between a cathode consisting of PMMA-coated CVD grown graphene on metal foil and a glassy carbon anode in an aqueous solution of potassium thiosulfate results in the electrolysis of water and the generation of hydrogen gas bubbles at the graphene-metal interface. These bubbles rapidly delaminate the graphene and polymer from the catalyst layer. This electrochemical technique, frequently termed 'bubbling transfer', is commonly employed for the 
transfer of CVD-grown graphene from copper foil substrates and other metals [7]. Graphene can also be electrochemically delaminated from copper catalyst layers by the application of a strong oxidizing potential, resulting in the dissolution of the catalyst, as demonstrated by Yang et al. [8]. This method is more controllable than chemical etching processes, but still results in the destruction of the catalyst layer.

Hydrogen bubbling transfer is readily applicable to graphene grown on pure copper foils, but can give undesirable results in more complicated catalyst systems, such as physical vapor deposited thin films on support substrates (for example, copper on oxidized silicon wafers), which show a tendency to delaminate at the catalyst-support interface rather than the graphene-catalyst interface [9].

The generation of hydrogen bubbles is also a concern for the integrity of the graphene film, since large surface tension forces encountered at gas-liquid interfaces are known to cause graphene to break and roll up - critical point drying is typically employed to avoid these effects [10-12], but is not applicable when the expanding bubbles are the driving force behind the delamination. Macroscopic tearing is a limiting factor for applications in both large area electrodes and electronics.

In this work, by applying a fixed potential of $-0.4 \mathrm{~V}$ versus an $\mathrm{Ag} / \mathrm{AgCl}$ reference electrode to the catalyst/graphene/polymer stack, we are able to delaminate the graphene/polymer from the copper catalyst without the production of hydrogen gas bubbles at more negative potentials, and avoiding dissolution of the catalyst at more positive potentials. Delamination of the graphene from the catalyst layer instead proceeds through the formation and removal of a layer of insoluble copper (I) oxide on the catalyst surface. This oxide layer grows in from the edge of the graphene due to 
dissolved oxygen in the electrolyte. The adhesion of the graphene to the catalyst layer is weakened, until the graphene delaminates - we therefore term this procedure oxidative decoupling transfer (ODT).

This transfer technique results in more than 94\% coverage of graphene over large areas (up to $100 \mathrm{~mm}$ in diameter), and also enables the use of deposited thin-films of copper catalyst without destructively delaminating such deposited catalyst films from their carrier substrates. The copper thin-films can be reused for growth after a short hydrogen annealing step (20 min) in the CVD process. Graphene regrown in this way shows comparable quality to the first graphene growth, as confirmed by Raman spectroscopy, demonstrated here by five repeated growth and transfer cycles applied to the same copper substrate without compromising the quality of the graphene film.

\section{Experimental}

\subsection{CVD growth}

Graphene is grown on either $\mathrm{Cu}$ foil (25 $\mu \mathrm{m}$ thick, Alfa Aesar, double side polished, 99.999\% purity) or a $1.5 \mu \mathrm{m}$ film of sputtered Cu supported by a 4” $\mathrm{SiO}_{2}(1 \mu \mathrm{m}) / \mathrm{Si}$ wafers by chemical vapor deposition (Aixtron Black Magic). The CVD of graphene on copper, which follows published recipes $[13,14]$, consists of an initial annealing phase (20 min) in a hydrogen/argon atmosphere and a growth phase (40 min), in which a methane precursor is introduced in the chamber.

A $5 \mu \mathrm{m}$ sacrificial layer of cellulose acetate butyrate (30 kDa, $0.3 \mathrm{~g} / \mathrm{l}$ in ethyl acetate) (CAB), is spun (4000 rpm, $60 \mathrm{~s}$ ) on the substrates after graphene growth, soft-baked at $80^{\circ} \mathrm{C}$ for 10 min and subsequently hard-baked at $130^{\circ} \mathrm{C}$ for an additional $10 \mathrm{~min}$. Graphene on the back side of the foils is removed by oxygen plasma (100 W, $2 \mathrm{~min})$. 


\subsection{Graphene transfer}

\section{Removal of Cu by etching}

Copper foils are etched away using ammonium persulphate (AP), following the procedure published in Refs [2, 15], which we summarise in Supplementary Information.

\section{Delamination of graphene-CAB by ODT method}

The copper/graphene/CAB stack is connected as the working electrode (WE) of an electrochemical cell. The counter electrode (CE) is a $200 \mathrm{~nm}$ thick layer of platinum sputtered onto a 4" oxidized silicon wafer to provide a large surface area. Picture and schematic of the system are reported in Figure S1. Voltages are applied to the WE by a potentiostat (Keithley 2400) with respect to a commercial Ag/AgCl reference electrode (RE), with current flowing only between the WE and CE. The electrolyte is $1 \mathrm{M}$ potassium chloride in deionized water, $\mathrm{pH}=5.5$. Local concentration gradients in the electrolyte are reduced by magnetic stirring.

\section{Adhesion to oxidized silicon}

The graphene/CAB stack is washed in deionized water and dried in a desiccator. The graphene surface is then placed onto an oxidized silicon wafer and dried at $80{ }^{\circ} \mathrm{C}$ for 1 hour. The temperature is increased to $135^{\circ} \mathrm{C}$ for two hours to soften the polymer and promote adhesion. After cooling, the sacrificial CAB layer is removed in ethyl acetate.

\subsection{Characterization}

\section{Raman Spectroscopy}

Raman spectra are recorded with a Thermo Fisher DXR microscope under ambient conditions using a $532 \mathrm{~nm}$ excitation laser source. The nominal spot size is $700 \mathrm{~nm}$. The power of the laser is kept 
below $1 \mathrm{~mW}$. We fit the Raman spectra with Lorentzian functions to extract peak height, width and position data.

\section{Optical microscopy}

We quantitatively measure the coverage of the transferred graphene using an analytical microscopy technique based on Ref [16] as well as gigapixel microscopy. A Nikon Eclipse L200 microscope equipped with a programmable Prior Scientific XYZ stage and a $10 \mathrm{x}$ objective with NA $=0.3$ ( $0.484 \mu \mathrm{m} /$ pixel) is used to acquire images with $20 \%$ overlap, allowing adjacent images to be stitched together. In order to minimize intensity variations each image was subject to $4 \mathrm{x}$ intensity averaging and dynamical autofocus. Unity gain was applied in the CCD sensor to minimize noise.

By converting the conventional wavelength-dependent contrast of graphene [16] into the corresponding contrast of the red, green, and blue (RGB) channels, the RGB contrasts of clean, single-layer graphene on $300 \mathrm{~nm} \mathrm{SiO}_{2}$ were found to be $\sim 8.1 \%$, 3.6\%, and $-0.2 \%$, respectively. $\mathrm{CAB}$ was found to have a contrast changing linearly with thickness for thin films with the presence of graphene simply serving as an offset. By allowing for small variations in the pixel values, the above information can be used to generate three unique sets of pixels, defining either residues, graphene (with or without residues), or oxide. Binary images of the samples can then be generated, where white indicates graphene and black indicates absence of graphene.

\section{Terahertz Spectroscopy}

Terahertz sheet conductance maps were obtained with a fiber-coupled terahertz time-domain spectrometer (THz-TDS). Transmission measurement of sub-picosecond, electromagnetic pulses through the graphene films, allows contact-free measurement of the complex sheet conductance as a function of frequency from 0.1 to $1.4 \mathrm{THz}$. This covers the lower range of the typical intraband (Drude) conductivity response of CVD graphene, where AC sheet conductance can be directly 
extrapolated to the DC sheet conductance value of the film [17]. By raster scanning the graphene films in a focused beam, sheet conductance maps were built from the average real sheet conductance from 0.9-1.0 THz, with a diffraction-limited, spatial resolution of approximately 350 $\mu \mathrm{m}$. Details of the experimental setup and data analysis method are described elsewhere [17].

\section{Electron Microscopy}

The SEM micrographs are taken in a Zeiss Supra $40 \mathrm{VP}$ Microscope at $5 \mathrm{kV}$ accelerating voltage with a current of $0.17 \mathrm{nA}$.

\section{$X$-ray Photoemission Spectroscopy}

The measurements are performed in ThermoScientific K-Alpha X-ray Photoelectron Spectrometer System. The base pressure in the analytical chamber is $10^{-9}$ Torr. The source is a monochromatic Al $\mathrm{K} \alpha \mathrm{X}$-ray radiation (1486 eV). The X-ray gun spot was adjusted to be $400 \mu \mathrm{m}$. The energy resolution of X-ray source was approximately $1 \mathrm{eV}$. Data analysis was performed using ThermoAvantage version 4.87 .

\section{Cyclic Voltammetry}

We perform our measurements using the same equipment and experimental set-up as described in Section $2 \mathrm{~b}$ above. The voltage was swept forward from an initial value of $-1 \mathrm{~V}$ to $+0.7 \mathrm{~V}$, and then swept in a reverse direction to $-2.0 \mathrm{~V}$ before sweeping forward to $-1 \mathrm{~V}$ to complete the cycle. A scan rate of $50 \mathrm{mV} / \mathrm{s}$ was used throughout. 


\section{Results}

Graphene is grown using published recipes on copper thin films sputtered on oxidized silicon carrier substrates (see Experimental section). After growth, a layer of cellulose acetate butyrate (CAB) polymer is spun on top of the graphene to support it after delamination. A combination of edge bead removal of the polymer and (oxygen) plasma ashing of the graphene is used to expose the catalyst layer and the edge of the graphene (Figure $1 \mathrm{f}$ ).

The copper is oxidised beneath the graphene layer by applying a potential of $-0.4 \mathrm{~V}$ to the catalyst vs. an $\mathrm{Ag} / \mathrm{AgCl}$ reference electrode in a $1 \mathrm{M} \mathrm{KCl}$ solution. The oxidation front of the copper proceeds controllably from the exposed edge of the graphene layer, towards the center (Figure $1 \mathrm{~b}$, $\mathrm{f}, \mathrm{g})$ at an average velocity of $24 \mathrm{~mm}$ per hour. Control experiments performed in degassed electrolyte protected from atmosphere by a nitrogen flow showed no oxidation, and the graphene did not delaminate. In samples processed in oxygenated electrolytes - those exposed to ambient conditions - a rise in the $\mathrm{pH}$ value from 3.5 to 8.0 is observed locally at the freshly exposed copper surface directly after delamination of graphene.

The delaminated CAB-coated graphene is then adhered to a target substrate and the CAB is removed in ethyl acetate solvent, similar to typical PMMA-based transfer techniques (see Experimental section). 


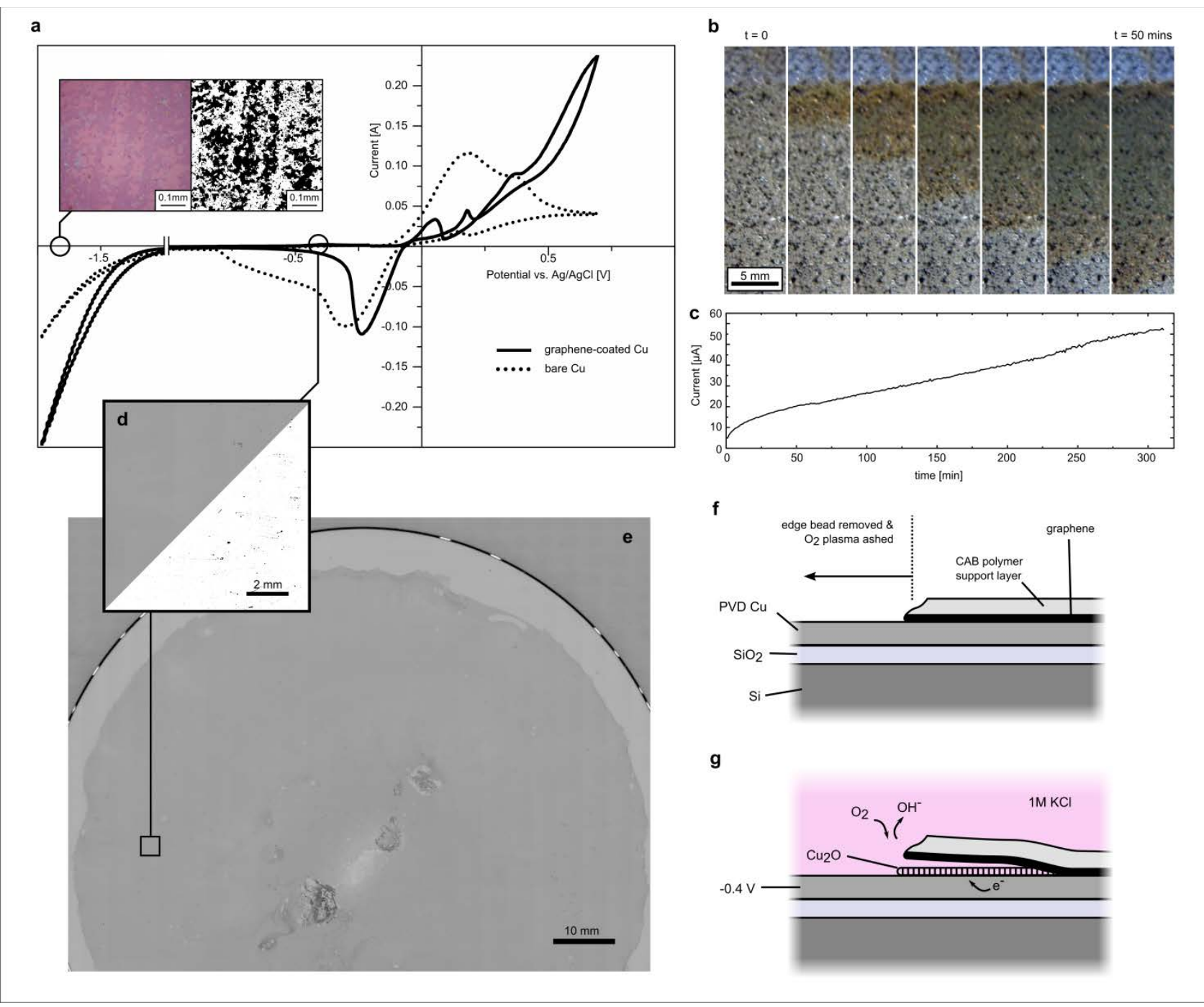

Figure 1. a) Cyclic voltammetry of copper and graphene coated copper foil. The voltage is swept between -2 $\mathrm{V}$ and $0.7 \mathrm{~V}$ (see Methods and Supplementary Information). Inset: Optical image and relative coverage map (average coverage of 48.7\%) of graphene transferred by bubbling (-2 V) b) Optical images of oxidation front progressing beneath graphene/CAB. c) Current vs. time from the counter to the working electrode during ODT transfer - sample size $4 \mathrm{~cm}^{2}$. d) Optical image of transferred graphene (left triangle) with superimposed map of coverage (right triangle). White pixels indicate expected graphene contrast. Total coverage of 99.6\% e) Gigapixel optical map of graphene transferred to $100 \mathrm{~mm}$ diameter $\mathrm{SiO}_{2}(100 \mathrm{~nm}) / \mathrm{Si}$ wafer. f) Sample schematic. Catalyst layer is exposed by edge bead removal of $\mathrm{CAB}$ and $\mathrm{O}_{2}$ plasma ashing of graphene. g) Applying -0.4 V vs. Ag/AgCl results in oxidation of the catalyst surface and delamination of graphene.

Figure 1 e shows an optical image of a $100 \mathrm{~mm}$ diameter region of single layer graphene transferred by ODT. We quantitatively measured the coverage of graphene produced on the silicon dioxide by digitally imaging the entire surface at a resolution of $0.96 \mu$ m per pixel, with a $20 \%$ oversampling to 
enable image stitching (see Experimental section). The resulting gigapixel image (Supplementary Information) shows the expected graphene contrast of $\sim 7 \%$ on $100 \mathrm{~nm}$ oxidized silicon in $94.5 \%$ of the pixels over the entire wafer surface - most of the non-covered oxide surface is localized in macroscopic areas (some $5-10 \mathrm{~mm}^{2}$ in size), and, outside of these areas, the graphene coverage is better than 99\% (Figure $1 \mathrm{~d}$ ).

After ODT of graphene and rinsing in DI water, the $\mathrm{Cu}$ thin-film substrate is re-used for CVD growth of graphene. The growth-transfer cycle was repeated for a total of 5 times. The transferred graphene is characterized with Raman spectroscopy by collecting more than 2500 point spectra per sample (Experimental section). Graphene films show consistent Raman 2D:G and D:G peak ratios when grown on recycled catalyst to which this method has been previously applied (Figure 2 a - c). In particular, the distributions of the D:G peak ratios (Figure 2 a) are largely centered around 0.1, with the exception of growth $4(\sim 0.22)$. The values of the 2D:G peak ratios (Figure $2 \mathrm{~b}$ ) range from a mean of $\sim 1.32$ for the first growth to $\sim 2$ for the fifth growth.

We compare the properties of graphene grown on PVD copper thin films and transfer by ODT to graphene obtained by the more common route of growth on copper foils and etching in ammonium persulphate. An identical growth recipe was used in both cases (see Experimental section). The ODT-transferred graphene generally shows a distribution of the Raman D:G peak ratio centered around 0.1 , as compared to 0.26 for graphene grown by the same recipe on copper foils and transferred by ammonium persulphate etching (Figure 2 a). 


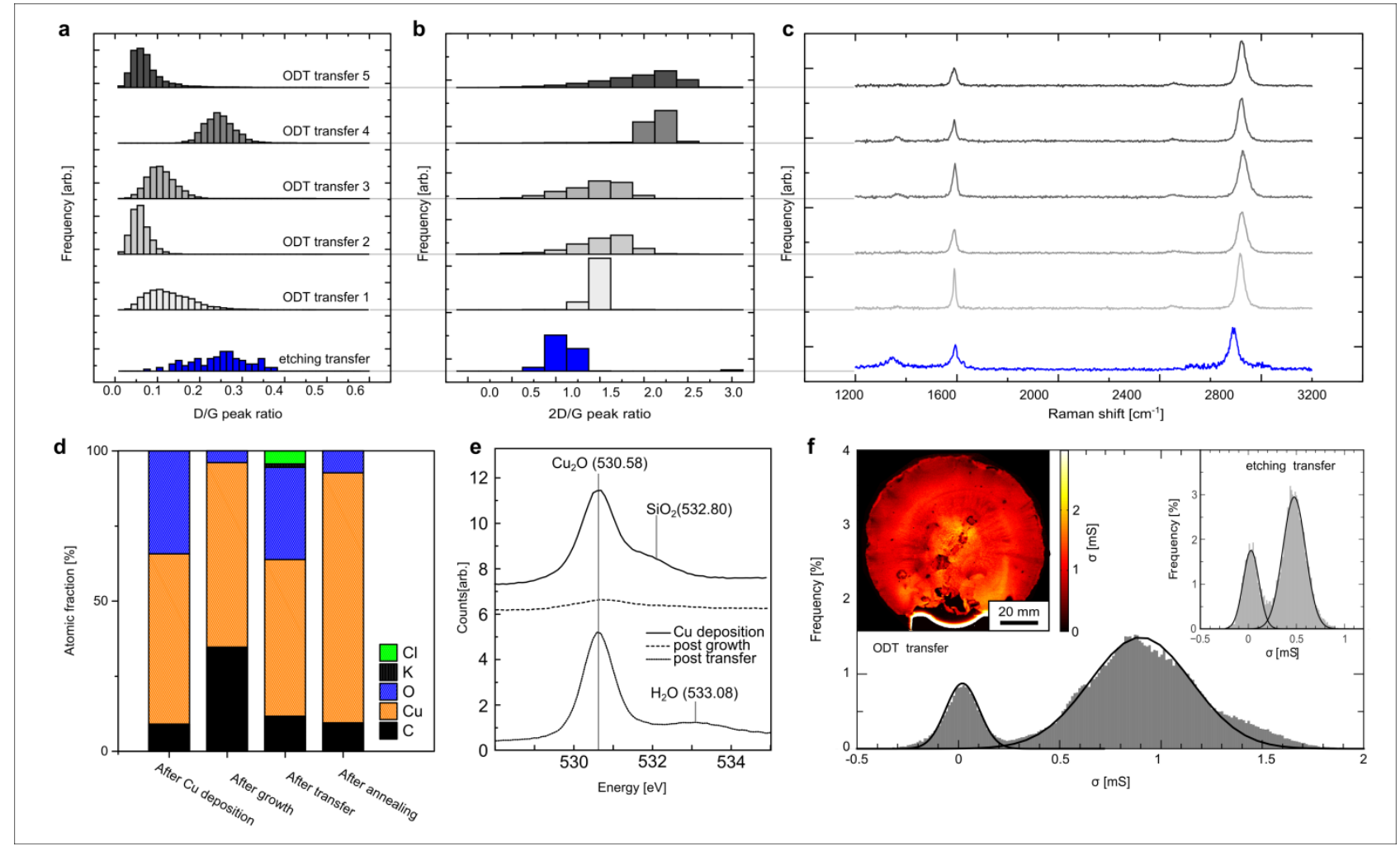

Figure 2. a, b) Bottom to top: Histograms of 2500 Raman D:G (a) and 2D:G (b) peak ratios collected from graphene grown on commercial $\mathrm{Cu}$ foil and transferred by etching of the catalyst (blue, bottom), and five consecutive growth-transfer cycles using ODT from a single PVD copper sample (greyscale).c) Examples of single raw Raman spectra from the ensemble used for the histograms in $(a, b)$. d) XPS analysis of the copper surface after copper deposition, after graphene growth, after ODT transfer $(-0.4 \mathrm{~V})$ and after water rinsing and annealing in the CVD chamber. e) The O1s peak measured by XPS. f) Histograms of average sheet conductance from 0.9 - $1.0 \mathrm{THz}$ with normal distribution peak fits of graphene grown on 4" $\mathrm{Si} / \mathrm{SiO}_{2}$ coated with PVD Cu film and transferred by ODT. Left inset shows the respective map. Right inset: Histograms of average sheet conductance from 0.9 - $1.0 \mathrm{THz}$ with normal distribution peak fits of conventional etching transfer of graphene from copper foils.

We use x-ray photoelectron spectroscopy (XPS) to determine the composition of the PVD deposited copper surface at each stage of our process (Figure $2 \mathrm{~d}$ ). XPS shows the presence of $\mathrm{Cu}_{2} \mathrm{O}$ on the copper surface before growth and after transfer - no copper (I) oxide can be detected after graphene growth. After transfer of the graphene from the copper, elemental analysis shows the presence of both $\mathrm{K}$ and $\mathrm{Cl}$, originating from the electrolyte. These species are removed by deionized water rinsing and annealing prior to regrowth to recover the original metallic copper surface. We 
determine the change in mass of individual catalyst layers plus carrier substrate at each stage of the growth and transfer process for 10 different growths using a quartz crystal microbalance - the sputtered copper catalyst weighs 122 +/- $22 \mathrm{mg}$ for a $100 \mathrm{~mm}$ diameter catalyst layer, corresponding to around $2000 \mathrm{~nm}$ of sputtered copper. A full cycle-results in an average loss of mass of $13.9 \mathrm{mg}+/-0.8 \mathrm{mg}$, a change in mass of around $11 \%$, which corresponds to $200 \mathrm{~nm}$ loss in thickness of the catalyst layer. This loss occurs almost exclusively after graphene growth as a result of the evaporation of copper at the low growth chamber pressure used (10 mbar). We were also unable to detect a change in mass or thickness of a bare copper catalyst layer after it was subjected to 100 hours of ODT conditions (fixed potential at $-0.4 \mathrm{~V}$ ).

We performed spatially resolved sheet conductance mapping of as-transferred graphene films obtained by both ODT (graphene grown on PVD copper, $100 \mathrm{~mm}$ diameter) and etching (commercial $\mathrm{Cu}$ foil) using terahertz time domain spectroscopic (THz-TDS) mapping [17]. The sheet conductance maps of the transferred films show homogenous conductance, except for the small number of mm-scale defects visible in Figure $2 \mathrm{f}$, left inset. Histograms of the two sheet conductance maps show bimodal distributions (Figure 2 f). Each sample histogram displays a Gaussian peak centered at $0 \mathrm{mS}$, which reflects the zero-conductance background of the areas not covered by graphene. In addition the graphene transferred by etching from foils and the graphene transferred by ODT from PVD film show Gaussian peaks at $0.5 \mathrm{mS}$ and $0.95 \mathrm{mS}$, respectively, reflecting the actual sheet conductance distribution for the transferred graphene films. A small shoulder is also visible at the higher conductance of 1.3-1.4 mS for the ODT-transferred graphene from PVD copper. The broadening of the peaks is due to spatial blurring near edges of the graphene films in addition to any inhomogeneity in graphene conductance related to the growth process. 


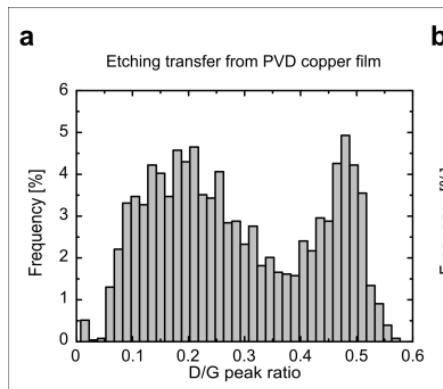

b
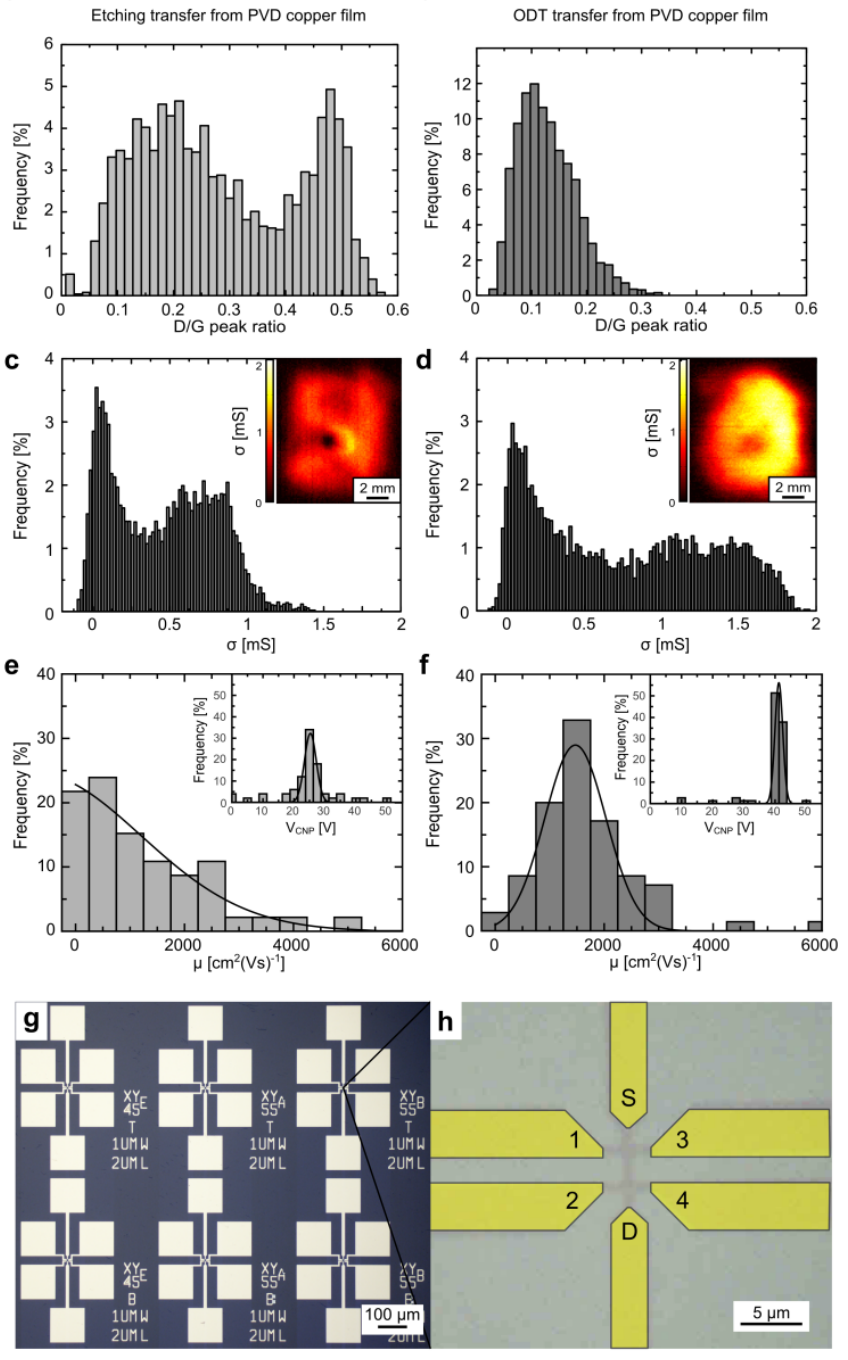

Figure 3. a, b) Histograms of Raman D:G peak ratios collected from graphene grown (a) on PVD Cu and transferred by etching and (b) by ODT transfer from PVD Cu. c, d) Histograms of THz spectroscopic map of sheet conductance of the same graphene sample analysed in $(a, b)$. Insets show the respective maps. e, f) Histograms of mobility measurements with normal distribution fits of graphene devices fabricated by (c) etching of PVD copper film and (d) ODT transfer from PVD copper film. Insets show histograms of charge neutrality points for the respective sets of devices. g) Optical image of G-FET devices. h) Optical image of graphene Hall bar defined in transferred graphene overlaid with schematic of contact geometry.

In order to characterize the effect of different transfer techniques on the electrical quality of graphene, we fabricated graphene field effect transistors (G-FET) with Hall bar geometries by electron beam lithography on $\mathrm{SiO}_{2}(300 \mathrm{~nm})$ / Si substrates (Figure $3 \mathrm{~g}$, h) from graphene grown on 
PVD copper films, using either ODT or etching in ammonium persulphate to transfer the graphene. More than 1280 individual G-FET devices were fabricated and electrically characterized (four point measurements) using an automatic probe station.

The fabrication yield was higher for graphene transferred by ODT (13.4\%) than for graphene delaminated by etching the catalyst layer in ammonium persulphate (9.6\%).

The average mobility of devices produced by ODT was $\mu=1930 \mathrm{~cm}^{2}(\mathrm{Vs})^{-1}$ with a standard deviation of $950 \mathrm{~cm}^{2}(\mathrm{Vs})^{-1}$, as compared to $1450 \mathrm{~cm}^{2}(\mathrm{Vs})^{-1}$ with a standard deviation of 1190 $\mathrm{cm}^{2}(\mathrm{Vs})^{-1}$ for graphene transferred by etching (Figure $3 \mathrm{e}, \mathrm{f}$ ). Moreover, etching transfer results in a distribution of measured mobility values with a long tail, rather than the normally distributed values for mobility observed for ODT. While the yield and the electrical quality in the case of ODT was noticeably better than the etching case, graphene samples transferred by etching display a lower level of p-doping, with $\mathrm{V}_{\mathrm{CNP}}=25.5 \mathrm{~V}$ as compared to $40.5 \mathrm{~V}$ for graphene transferred by electrochemical delamination, but etching transfer results in a larger spread of observed doping levels, with a standard deviation of $8.8 \mathrm{~V}$ vs. $6.1 \mathrm{~V}$ (Figure $3 \mathrm{e}, \mathrm{f}$ insets), indicating that the ODT results in more reproducible and stable device performance.

We further investigate graphene grown on PVD copper films and transferred using either ODT or etching in ammonium persulphate by Raman and THz spectroscopy. The histograms of the D:G peak ratios of graphene grown on PVD film and transferred by ODT are normally distributed around 0.1 , with only few values above 0.2 (Figure 3 a). In contrast, the etched PVD samples show a bimodal distribution of $\mathrm{D}: \mathrm{G}$ peak ratios, with two peaks at 0.2 and 0.5 (Figure $3 \mathrm{~b}$ ). As reported in Figure $3 \mathrm{c}$, d, the distributions of the THz sheet conductances are both bimodal, with a peak at $0 \mathrm{mS}$ (zero-conductance peak), and show higher values on average for graphene transferred by ODT (distribution centered around $1.3 \mathrm{mS}$ ) in comparison to the film transferred by etching of the PVD copper (centered around $0.8 \mathrm{mS}$ ). 


\section{Discussion}

Copper exposed to dissolved oxygen in the electrolyte naturally forms copper (I) oxide, $\mathrm{Cu}_{2} \mathrm{O}$ :

$4 \mathrm{Cu}+\mathrm{O}_{2} \rightarrow 2 \mathrm{Cu}_{2} \mathrm{O}$

The volume increase and binding energy change associated with this oxidation decouples the graphene from the catalyst surface [18]. In the absence of an externally applied potential, copper (I) oxide formed at the edge of the graphene will passivate the catalyst by preventing exposure to dissolved oxygen and further oxidation. The reaction would thus terminate with this stable oxide. Application of $-0.4 \mathrm{~V}$ vs. $\mathrm{Ag} / \mathrm{AgCl}$ in an aqueous solution is sufficient to reduce the copper (I) oxide back to metallic copper, producing hydroxide ions:

$\mathrm{Cu}_{2} \mathrm{O}+\mathrm{H}_{2} \mathrm{O}+2 e^{-} \rightarrow 2 \mathrm{Cu}+2 \mathrm{OH}^{-}$

In our case this results in progress of the oxidation front beneath the graphene and polymer stack, leaving an excess of hydroxide ions. Capillary forces draw the electrolyte further under the graphene layer, eventually resulting in delamination. This mechanism would account for our observations that degassed electrolytes are ineffective - preventing the continued formation of copper (I) oxide - and that a locally more basic $\mathrm{pH}$ is observed at the area where graphene delaminates from copper.

The copper oxidation process described here reduces the binding energy of the graphene to the catalyst layer, resembling an intercalation process [19]. In combination with growth on sputtered catalyst layers we achieve extremely high coverages of graphene during transfer - over $94 \%$ across a $100 \mathrm{~mm}$ diameter wafer. Coverages are substantially higher than those which we achieve by hydrogen bubbling (as shown in Figure 1 a, inset) and comparable with those we obtain by complete dissolution of the copper catalyst layer (Supplementary Information). We ascribe the 
extremely high graphene coverages observed to a lack of gas-liquid interfaces encountered by the graphene during this process - suspended graphene is likely to break due to the surface tension forces at such interfaces. Critical point drying is often used to avoid such effects in the preparation of these samples [10-12].

We note that similar measures of coverage are not available for previous studies, due to the difficulty in determining coverage accurately over large areas prior to the presentation of the gigapixel optical microscopy techniques described here.

Although microscopic tearing is reduced, macroscopic defects are visible in Figure 1 e due to pockets of trapped air during placement of the graphene/CAB stack on the target substrate. These pockets prevent adhesion of the graphene to the target substrate, and result in locally incomplete transfer. These problems are also encountered when transferring graphene from copper foils, which are prone to plastic deformation on handling leading to a poor contact when transfer to flat substrates is attempted. These effects are enhanced by the thick polymer layer used in our transfers. However, the use of a thick polymer layer is justified by the necessity of having a large and mechanically stable support layer able to withstand the forces inside the electrochemical cell. Such a thick polymer layer allows for easy handling and simplifies the drying of graphene after water rinsing [20].

The consistency of the 2D:G and D:G peak ratios over the five repeated growth and transfer cycles demonstrates the preservation of the Cu catalyst layer during ODT transfer. In our case, it is the loss of catalyst mass through evaporation during low pressure CVD which limits the reuse of the catalyst, and not the transfer technique demonstrated. Evaporation of a mass corresponding to approximately $200 \mathrm{~nm}$ of catalyst across a $100 \mathrm{~mm}$ wafer surface was observed during our experiments, which could be reduced by increasing the growth pressure. We were unable to observe 
any dissolution of copper during exposure of a bare copper surface to ODT conditions for a period of 100 hours.

Improvements in the D:G peak ratios, which correspond to a reduce density of defects [21], observed for graphene transferred by ODT from PVD copper over those transferred by etching from copper foils (Figure 2 a) can be ascribed to a combination of both the reduced damage induced by the transfer process and a lack of residual catalyst particles from etching-based transfer. Such particles can gather at edges and defects and provide an enhancement of the Raman D peak signal from these areas. [22-24]. This interpretation is also supported by our observations that graphene grown on PVD films and transferred by etching shows a marked increase in D:G peak ratio over graphene from the same PVD films transferred by ODT (Figure $3 \mathrm{a}, \mathrm{b}$ ).

THz-TDS measurements show that the sheet conductance for graphene grown on PVD copper films and transferred by ODT is relatively homogeneous, especially if compared to previously published results [17]. Higher values of the sheet conductance visible as the shoulder in the distribution (Figure $2 \mathrm{f}$ ) originate from localized areas of the sheet, particularly in the center (Figure $2 \mathrm{f}$, left inset). We ascribe this difference to temperature inhomogeneity during growth in our CVD system - higher temperatures in the central area of the catalyst wafer may give rise to graphene with slightly higher electrical conductance here. Such observations demonstrate how THz-TDS mapping could be used to non-destructively probe the large-scale conductance of transferred graphene layers.

The higher mobility values observed for devices produced from graphene using ODT show that the growth of graphene on PVD films and transfer by this technique is a promising route for the production of devices. We ascribe this difference to a lack of contamination from residual catalyst particles and etchant residues when applying ODT transfer. Although potassium present in the electrolyte is a strong n-dopant of graphene [25], strong p-type doping is present in devices 
produced by etching and ODT techniques. p-doping is typically observed for graphene FET devices exposed to ambient conditions and without pretreatment of the silicon oxide surface [26]. Annealing of the devices to remove contaminants or treatment of the silicon dioxide surface to make it more hydrophobic [26] would reduce the observed doping. We note that in both datasets there are outlier devices which demonstrate high calculated mobility values up to $\sim 12,000$ $\mathrm{cm}^{2}(\mathrm{Vs})^{-1}$. These results are also supported by THz measurements on the same films, which show an average sheet conductance higher for graphene transferred by ODT than by etching from the same PVD copper samples (Figure 3 c, d).

\section{Conclusions}

We have demonstrated the transfer of $100 \mathrm{~mm}$ diameter graphene layers from sputtered layers of copper catalyst by the introduction of a new transfer technique based on the oxidative decoupling of graphene from its growth substrate, resulting in graphene coverage greater than $94 \%$ across a wafer surface, as demonstrated by quantitative wafer-scale gigapixel optical microscopy mapping of the contrast. The use of a reducing electrochemical potential $(-0.4 \mathrm{~V})$ applied with respect to a reference electrode prevents the dissolution of the copper catalyst, and enables the continual formation and reduction of a copper (I) oxide layer on the catalyst surface, which delaminates the graphene. In combination with a hydrophobic polymer support (CAB), this lifts the graphene from the catalyst surface with minimal mechanical and electrical damage.

Graphene field effect transistors produced using this technique demonstrate a mobility 30\% higher than those produced from the same graphene but delaminated by dissolving the catalyst in ammonium persulphate. Though ODT method leads to a higher level of p-doping, it provides improved consistency and yield. 
The ODT technique demonstrated is applicable to thin film catalyst layers which would be destroyed by previously published chemical etching or hydrogen bubbling techniques. It is not possible to apply the hydrogen bubbling electrochemical transfer technique to thin film catalyst layers on carrier substrates that are the subject of this investigation, as hydrogen evolution at the catalyst / carrier substrate interface delaminate and destroy the catalyst layer rather than delaminating the graphene. The presented ODT technique is to our knowledge the only means of transferring graphene from thin copper films supported by carrier substrates whilst simultaneously enabling the reuse of the catalyst film.

The use of thin film catalysts is advantageous because it reduces the amount of copper catalyst needed for the growth of graphene to around 0.1 g per $100 \mathrm{~mm}$ diameter wafer, and the enhanced flatness of the wafer surface allows closer contact of the graphene-polymer stack to a target wafer surface during transfer, improving coverage. Additionally, the purity of sputtered copper layers can be controlled at source, and the layers can be produced under cleanroom conditions to minimize surface contamination. Thinner copper layers also require shorter annealing times, improving the throughput of the CVD process.

Our results show that it is possible to reuse such thin catalyst layers multiple times without loss of quality in the grown and transferred graphene. We succeeded in reusing a catalyst layer four times above and beyond the initial growth, with reuse of catalyst layers limited only by loss of catalyst through evaporation of $\mathrm{Cu}$ during growth. Any loss of catalyst mass could also be replaced by a remedial catalyst sputtering step, as opposed to the complete replacement of the catalyst layer necessary if it is completely dissolved. Moreover, atmospheric pressure CVD processes would reduce the metal evaporation during graphene growth, resulting in enhanced reusability of the catalyst. 
The non-destructive delamination of graphene and recycling of the copper catalyst demonstrated here also allows the use of highly optimized growth substrates such as single crystal copper ingots for CVD growth. The very high coverage levels observed over large areas open the way for waferscale graphene device production from CVD graphene grown on reusable, highly optimized catalyst layers, whilst minimizing costs and maximizing graphene quality.

\section{Acknowledgments}

F.P. gratefully acknowledges support from the Danish Council for Independent Research, Technology and Production Sciences grant G-MOL. T.B. gratefully acknowledges support from the Danish Council for Independent Research, Technology and Production Sciences grant GraVOX. T.B. and P.B. gratefully acknowledge support from the Danish National Research Foundation Center of Excellence for Nanostructured Graphene, project DNRF58. S. L. acknowledges support from the Qualcomm Innovation Fellowship (QInF) 2013 and AFOSR MURI FA9550-09-1-0705. 


\section{References}

[1] Li X.; Cai W.; An J.; Kim S.; Nah J.; Yang D.; et al. - Large-area synthesis of high-quality and uniform graphene films on copper foils. Science 2009; 324(5932), 1312-1314.

[2] Bae S.; Kim H.; Lee Y.; Xu X.; Park J.-S.; Zheng Y.; et al. - Roll-to-roll production of 30-inch graphene films for transparent electrodes. Nat. Nanotech. 2010; 5, 574-578.

[3] Li X.; Zhu Y.; Cai W.; Borysiak M.; Han B.; Chen D.; et al. - Transfer of large-area graphene films for high-performance transparent conductive electrodes. Nano Lett. 2009; 9(12), 4359-4363.

[4] Kang J.; Shin D.; Bae S.; Hong B. H. - Graphene transfer: key for applications. Nanoscale 2012; 4, 5527-5537.

[5] Xu-Dong Chen X.-D.; Liu Z.-B.; Zheng C.-Y.; Xing F.; Yan X.-Q.; Chen Y.; Tian J.-G. High-quality and efficient transfer of large-area graphene films onto different substrates. Carbon 2013; 56, 271-278.

[6] Wang Y.; Zheng Y.; Xu X.; Dubuisson E.; Bao Q.; Lu J.; Loh K. P. - Electrochemical delamination of CVD-Grown graphene film: toward the recyclable use of copper catalyst. ACS Nano 2011; 5 (12), 9927-9933.

[7] Gao L.; Ren W.; Xu H.; Jin L.; Wang Z.; Ma T.; et al. - Repeated growth and bubbling transfer of graphene with millimetre-size single-crystal grains using platinum. Nat. Comm. 2012; 3, 699.

[8] Yang X.; Peng H.; Xie Q.; Zhou Y.; Liu Z. - Clean and efficient transfer of CVD-grown graphene by electrochemical etching of metal substrate. J. Electroanalyt. Chem. 2013; 688, 243248.

[9] de la Rosa C. J. L.; Sun J.; Lindvall N.; Cole M. T.; Nam Y.; Löffler M.; et al. - Frame assisted $\mathrm{H}_{2} \mathrm{O}$ electrolysis induced $\mathrm{H}_{2}$ bubbling transfer of large area graphene grown by chemical vapor deposition on Cu. Appl. Phys. Lett. 2013; 102, 022101. 
[10] Booth T. J.; Blake P.; Nair R. R.; Jiang D.; Hill E. W.; Bangert U.; et al. - Macroscopic graphene membranes and their extraordinary stiffness. Nano Lett. 2008; 8(8), 2442-2446.

[11] Bolotin K. I. ; Sikes K. J.; Jiang Z.; Klima M.; Fudenberg G.; Hone J.; et al. - Ultrahigh electron mobility in suspended graphene. Sol. State Comm. 2008; 146, 9-10, 351-355.

[12] van der Zande A. M.; Barton R. A.; Alden J. S.; Ruiz-Vargas C. S.; Whitney W. S.; Pham P. H. Q.; et al. - Large-scale arrays of single-layer graphene resonators. Nano Lett. 2010; 10(12), 4869-4873.

[13] Tao L.; Lee J.; Holt M.; Chou H.; McDonnell S. J.; Ferrer D. A.; et al. - Uniform wafer-scale chemical vapor deposition of graphene on evaporated $\mathrm{Cu}(111)$ film with quality comparable to exfoliated monolayer. J. Phys. Chem. C 2012; 116(45), 24068-24074.

[14] Chen S.; Ji H.; Chou H.; Li Q.; Li H.; Suk J. W.; et al. - Millimeter-size single-crystal graphene by suppressing evaporative loss of $\mathrm{Cu}$ during low pressure chemical vapor deposition. Adv. Mat. 2013; 25(14), 2062-2065.

[15] Suk J. W.; Kitt A.; Magnuson C. W.; Hao Y.; Ahmed S.; An J.; et al.- Transfer of CVD-grown monolayer graphene onto arbitrary substrates. ACS Nano 2011; 5(9), 6916-6924.

[16] Blake P. ; Hil, E. W.; Castro Neto A. H. ; Novoselov K. S.; Jiang D.; Yang R.; et al. - Making graphene visible. Appl. Phys. Lett. 2007; 91, 063124.

[17] Buron J. D.; Petersen D. H.; Bøggild P.; Cooke D. G.; Hilke M.; Sun J.; et al. - Graphene conductance uniformity mapping. Nano Lett. 2012; 12(10), 5074-5081.

[18] Kidambi P.R.; Bayer B.C.; Blume R.; Wang Z.-J.; Baehtz C.; Weatherup R.S.; et al. Observing Graphene Grow: Catalyst-Graphene Interactions during Scalable Graphene Growth on Polycrystalline Copper. Nano Lett. 2013; 13, 4769-4778

[19] Lu A.-Y.; Wei S.-Y.; Wu C.-Y.; Hernandez Y.; Chen T.-Y.; Liu T.-H.; et al. - Decoupling of CVD graphene by controlled oxidation of recrystallized Cu. RSC Advances 2012; 2, 3008-3013. 
[20] Petrone N.; Dean C. R.; Meric I.; van der Zande A. M.; Huang P. Y.; Wang L.; et al. Chemical vapor deposition-derived graphene with electrical performance of exfoliated graphene. Nano Lett. 2012; 12, 6, 2751-2756.

[21] Ferrari A.C. Raman spectroscopy of graphene and graphite: disorder, electron-phonon coupling, doping and nonadiabatic effects. Sol. State Comm. 2007, 143(1-2), 47-57.

[22] Hua Ni Z.; Wang H. M.; Ma Y.; Kasim J.; Wu Y. H.; Shen Z. Y. - Tunable stress and controlled thickness modification in graphene by annealing. ACS Nano 2008; 2, 5, 1033-1039. [23] Iqbal M. W.; Kumar Singh A.; Iqbal M. Z.; Eom J. - Raman fingerprint of doping due to metal adsorbates on graphene. J. Phys.: Condens. Matter 2012; 24, 335301.

[24] Schedin F.; Lidorikis E.; Lombardo A.; Kravets V. G.; Geim A. K.; Grigorenko A. N.; et al. Surface-Enhanced Raman Spectroscopy of Graphene. ACS Nano 2011; 4, 10, 5617-5626. [25] Bianchi M.; Rienks E. D. L.; Lizzit S.; Baraldi A.; Balog R.; Hornekær L.; Hofmann P. Electron-phonon coupling in potassium-doped graphene: angle-resolved photoemission spectroscopy. Phys. Rev. B 2010; 81, 041403.

[26] Lafkioti M.; Krauss B.; Lohmann T.; Zschieschang U.; Klauk H.; Klitzing K.; et al. Graphene on a Hydrophobic Substrate: Doping Reduction and Hysteresis Suppression under Ambient Conditions. Nano Lett. 2010; 10, 1149-1153. 